\title{
DESIGN AND NOISE OPTIMIZATION OF RF LOW NOISE AMPLIFIER FOR IEEE STANDARD 802.11A WLAN
}

\author{
Ravinder Kumar ${ }^{1}$, Munish Kumar², and Viranjay M. Srivastava ${ }^{1}$ \\ ${ }^{1}$ Department of Electronics and Communication Engineering, \\ Jaypee University of Information Technology, Solan-173234, India. \\ viranjaydieee.org \\ ${ }^{2}$ Department of Electronics and Communication Engineering, \\ Guru Jambheshwar University of Science and Technology, Hisar-125001, India
}

\begin{abstract}
Low noise amplifier is the front end block of radio-frequency receiver system. Its design required various characteristics such as power gain, noise figure, insertion losses and power consumption. In this paper we have proposed a single stage low noise amplifier design with high gain and low noise using inductive source degeneration topology for frequency range of $3 \mathrm{GHz}$ to $7 \mathrm{GHz}$ and also use the active biasing devices. A range of devices like inductors and capacitors are used to achieve $50 \Omega$ input impedance with a low noise factor. The design process is simulated process is using Advance Design System (ADS) and implemented in TSMC $0.18 \mu \mathrm{m}$ CMOS technology. A single stage low noise amplifier has a measured forward gain $25.4 \mathrm{~dB}$ and noise figure $2.2 \mathrm{~dB}$ at frequency $5.0 \mathrm{GHz}$.
\end{abstract}

\section{KEYWORDS}

Advanced design system, Low noise amplifier, Radio-frequency, Noise figure, Wireless network, CMOS, RF switch, VLSI.

\section{INTRODUCTION}

The low noise amplifiers (LNA) are key components in the receiving end of the communication system. Its performance is measured in a number of figures which are most notable while dynamic range, return loss and stability are examples of others. Signal received from the antenna is directly given to the low noise amplifier then external as well as internal noise of the circuit is reduced. In reference [1] two MOSFETs are used in cascode topology which has little effects with each other. Therefore, the device parameters of these two MOSFETs in cascode topology can be design separately, with almost no trade-off $[2,3]$. But this topology requires more area with complexity.

References [4-6] presented that LNA which are able to provide gain to input signal powers up to $15 \mathrm{dBm}$ without degrading linearity or adding much noise. Such properties enable the wireless receiver to operate in hostile communication environments. In low noise amplifier design, the most important factors are forward gain, low noise, matching and stability. In this paper, we design a CMOS LNA using single stage inductive source degeneration topology with respect to impedance matching and noise optimization. Generally the cascoded topologies are used in the 
designing of CMOS LNA. Here we have used the inductive degeneration common source topology with active device biasing. The use of active devices increases the temperature sensitivity. Major applications of the LNAs [7-9] are to increase the signal power as well as minimizing the noise. Srivastava et. al. [10] have proposed a model of the double-gate CMOS for double-pole four-throw RF switch design at 45-nm technology, which is also an application for the LNA. The proposed LNA operates at $5.0 \mathrm{GHz}$ frequency used in IEEE standard 802.11a WLAN.

\section{Low NoISE AMPLIFIER CirCUIT SyNTHESIS}

Selection of the devices is an important task for designing process, because the noise and impedance matching conditions are highly related to each other. In reference [11-13], a commongate topology has been used to realize wide-band characteristics but the common-gate topology usually has related higher noise figure (NF) than common-source topology. We have used a single stage inductive source degeneration topology as shown in Fig. 1, which uses only one MOSFET with source degeneration.

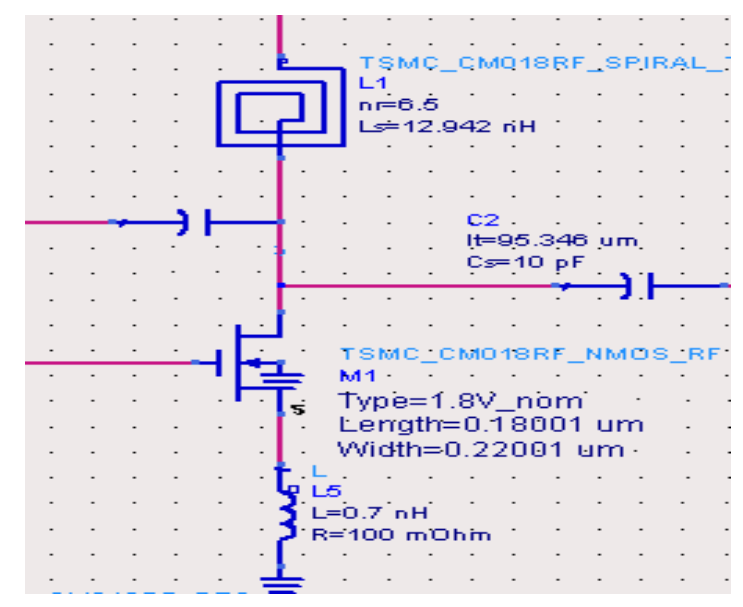

Figure 1. Single stage inductive source degeneration LNA.

The minimum NF can be made independent of the transistor width by selecting the source degeneration reactance [14]. The input impedance is given by:

$$
Z_{\text {in }}=\frac{V_{\text {in }}}{I_{\text {in }}}=S\left(L_{G}+L_{s}\right)+\frac{1}{s C_{g s}}+\frac{g_{m} L_{s}}{C_{g s}}
$$

when we use $50 \Omega$ impedance then Ls is solved as:

$$
L_{s}=\frac{C_{g^{s}}}{g_{m}} Z_{o}
$$

The LNA design formula and equation were referred to [15]. A low-noise amplifier is designed using a device's noise modal or noise parameters and S-parameters [16]. In a low noise amplifier, the transistor's input is matched for optimum noise figure and the transistor's output is conjugate matched with $50 \Omega$ system impedance for a maximum gain. The output noise of a two-port network with noise figure is given by:

$$
N_{o}=F G K T B
$$

If minimum detectable input signal is $\mathrm{X} \mathrm{dB}$ above the noise floor, then

$$
\begin{aligned}
& P_{\text {in }}^{\min }=N_{o}-G+X \\
& P_{\text {out }}^{\min }=N_{o}+X
\end{aligned}
$$




\section{DC BIASING}

The proper bias or quiescent point for the application to maintain constant current over transistor parameter variations is required due to the process and temperature. The most critical device parameter commonly used in biasing is $I_{\mathrm{dss}}$, the saturated current at zero gate bias. The change of $I_{D}$ with $V_{G}$ voltage is transconductance and is given by:

$$
g_{m}=\left.\frac{\Delta I_{D}}{\Delta V_{G}}\right|_{V_{d s}}
$$

Generally the active or passive type biasing is used. In this paper, we also used active component for biasing at $2.14 \mathrm{~V}$ and DC biasing network shown in Fig. 2.

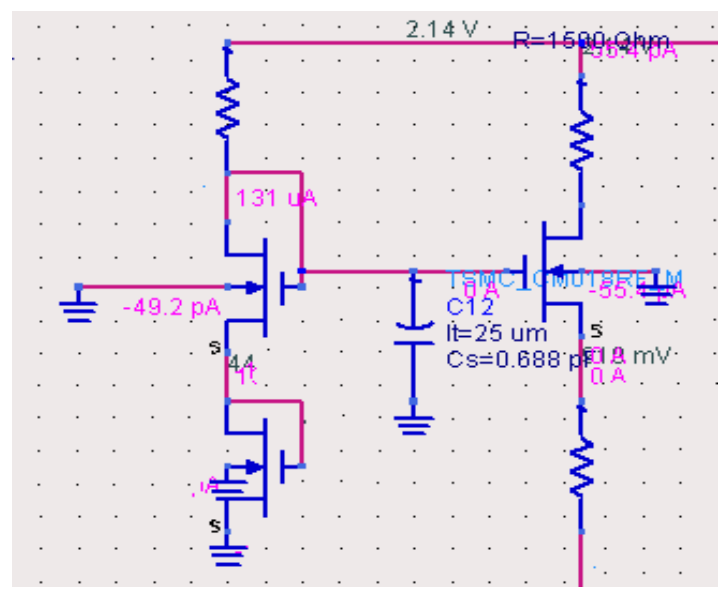

Figure 2. Active device DC biasing network.

\section{Single Stage Amplifier}

The amplifier consists of a transistor, the circuit for matching the source termination and the transistor, and that for matching the load termination and the transistor. The source and load terminations are usually $50 \Omega$. The useful gain to design an amplifier is transducer power gain, which accounts both source and load mismatching. The function of the matching circuits is to provide suitable source and load conditions for the transistor such that the transistor can generate power gain for the input signal under a stable condition which is shown in Fig. 1. Matching occurs when $Z\left(j \omega_{o}\right)=R_{s}$.

$$
\begin{array}{r}
\left(L_{G}+L_{S}\right) W_{o}=\frac{1}{W_{o} C_{g s}} \\
R_{S}=\frac{g_{m} L_{s}}{C_{g s}}
\end{array}
$$

where $R_{s}$ is the resistor associated in the input voltage source. LNA circuit of Fig. 1 is implemented in $0.18 \mu \mathrm{m}$ CMOS technology and then characterized. 
International Journal of VLSI design \& Communication Systems (VLSICS) Vol.3, No.2, April 2012

\subsection{Noise Figure}

The total noise power consists of amplified input noise entering the amplifier and the noise generated in the amplifier itself. The total noise bandwidth (NBW) is selected to make the total noise power correctly as:

$$
N B W=\frac{\int_{0}^{\infty} T_{A}(w) \cdot G_{A}(w) d w}{T_{A} \cdot G_{A}}
$$

The range of ' $w$ ' is limited by other components in the system, or by the gain response of the amplifier. The noise figure is given as:

$$
F=F_{\min }+\frac{R_{n}}{G_{s}}\left(G_{s}-G_{o p t}\right)^{2}
$$

The total equivalent input current noise is the sum of the reflected drain noise contribution and the induced gate current noise. Other parameter of two-port noise is shown as:

$$
G_{u}=\frac{i_{u}^{2}}{4 K T \Delta f}
$$

And the minimum noise figure is given by

$$
F_{\min }=1+2 R_{n}\left(G_{o}+G_{\text {opt }}\right)
$$

If there were no gate current noise, the minimum noise figure would be $0 \mathrm{~dB}$.

\subsection{Stability}

The stability of a circuit is characterized by stern stability factor $(\mathrm{K})$, Circuit is stable when $\mathrm{K}>1$ and $\Delta<1$.

$$
\begin{gathered}
K=\frac{1+\Delta^{2}-S_{11}^{2}-S_{22}^{2}}{2 S_{11} S_{22}} \\
\Delta=S_{11} S_{22}-S_{12} S_{21}
\end{gathered}
$$

Absolute stability is then determined when the input reflection coefficient $\Gamma_{\text {in }}<1$ and output reflection coefficient $\Gamma_{\text {out }}<1$. This is a very important consideration in a design and can be determined from the S-parameters, matching networks, and terminations [17].

\section{Matching Network}

To design the input and output impedance matching to maximize the power transfer and minimize the reflections we required the idea of matching network [18]. According to maximum power transfer theorem, the maximum power will be delivered to the load when the impedance of load is equal to the complex conjugate of the impedance of source means $Z_{s}=Z_{L}{ }^{*}$. The Smith chart is used to visualize the interactive process of impedance matching. Impedance matching network are shown in Fig. 3. Conjugate matching will satisfy the optimal noise condition and impedance matching simultaneously as a result of transistor sizing and bias condition. The ccomparison of LNA performance with other published data is shown in Table 1. 
International Journal of VLSI design \& Communication Systems (VLSICS) Vol.3, No.2, April 2012

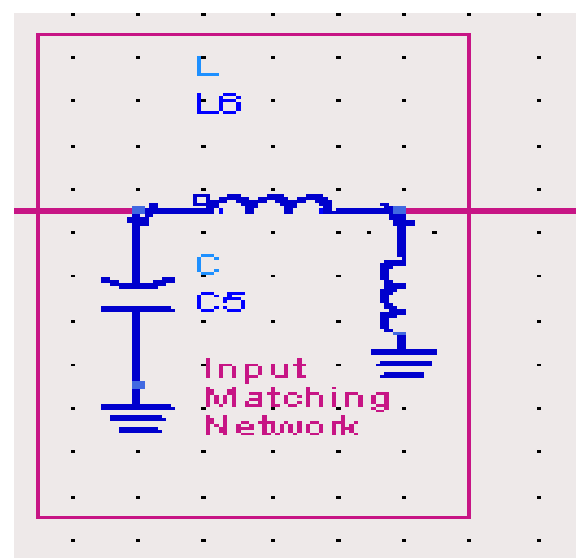

(a)

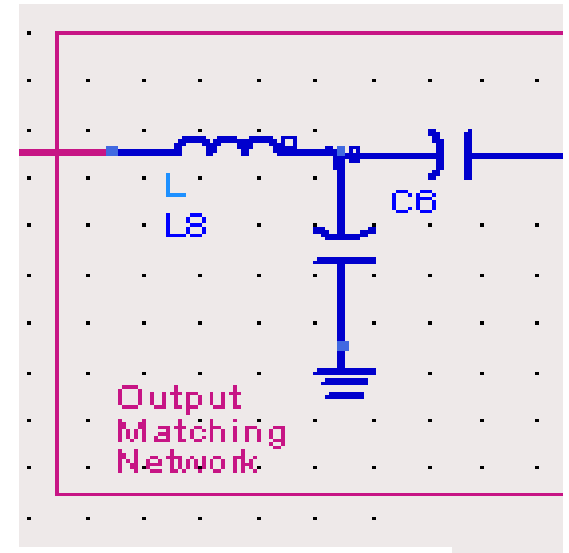

(b)

Figure 3. (a) Input and (b) Output impedance matching network.

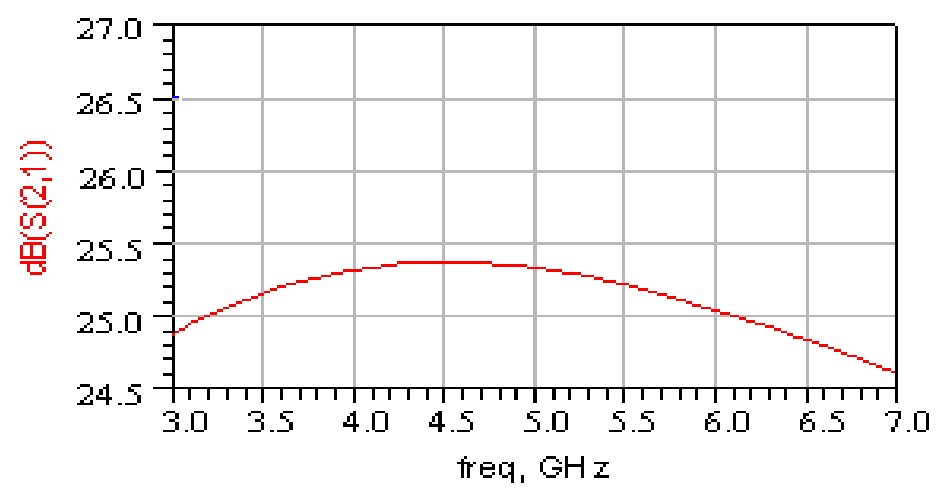

Figure 4. Simulated $S_{21}$ forward power gain at the frequency $5.0 \mathrm{GHz}$.

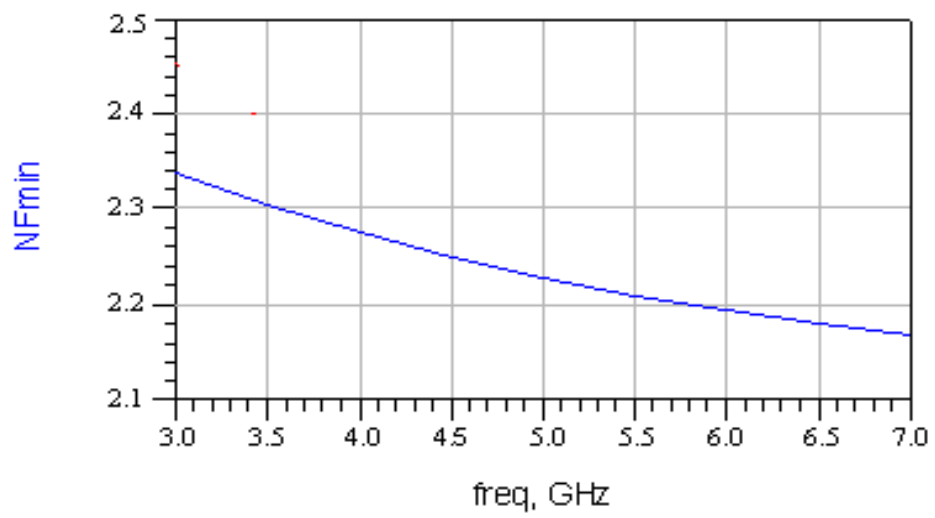

Figure 5. Simulated noise figure at 5.0 GHz. 
International Journal of VLSI design \& Communication Systems (VLSICS) Vol.3, No.2, April 2012

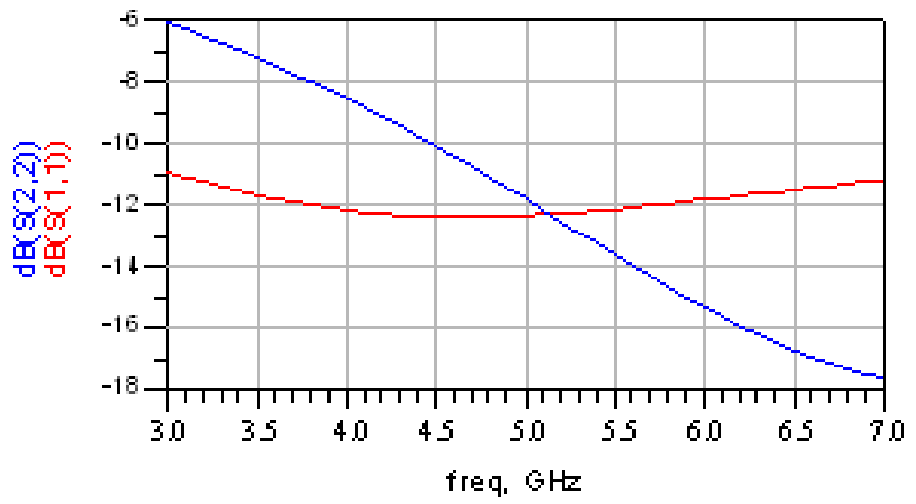

Figure 6. Simulated input and output return losses.

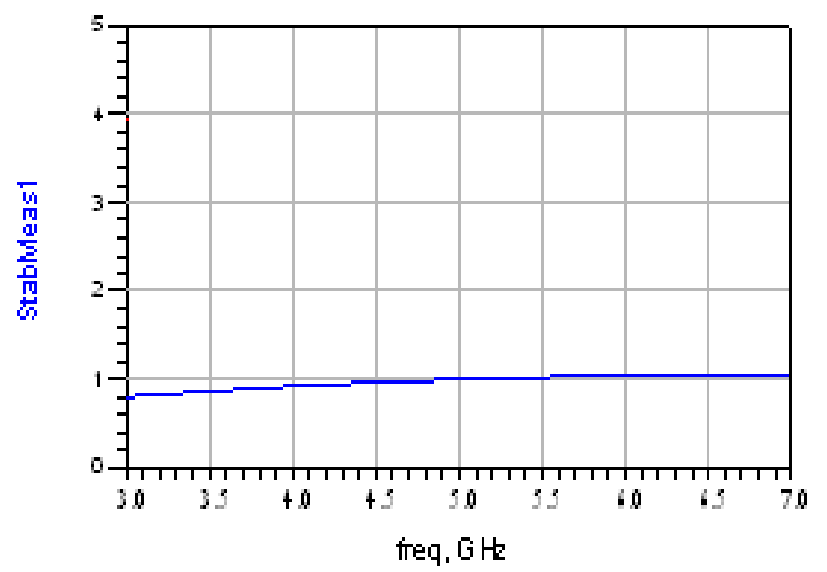

Figure 7. Stability factor vs. frequency.

\section{Simulation RESUltS AND CONCLUSIONS}

The designed LNA with matching network at $5.0 \mathrm{GHz}$ is obtained. The proposed LNA is implemented in TSMC $0.18 \mu \mathrm{m}$ CMOS technology. The simulation recorded that the amplifier gain $S_{21}$ is $25.3 \mathrm{~dB}$. The input insertion loss $S_{11}$ is $-12.2 \mathrm{~dB}$, overall noise figure (NF) is $2.2 \mathrm{~dB}$ and the output insertion loss $S_{22}$ is $-12 \mathrm{~dB}$. The best impedance matching for the high power gain and low noise in frequency range $3 \mathrm{GHz}$ to $7 \mathrm{GHz}$ shown in Fig. 4 to Fig. 7. Here a low noise amplifier circuit design for frequency of $3 \mathrm{GHz}$ to $7 \mathrm{GHz}$ has been presented and circuit simulations were done in ADS. LNA with $25.3 \mathrm{~dB}$ gain and $2.2 \mathrm{~dB}$ noise figure at frequency $5 \mathrm{GHz}$ to $6 \mathrm{GHz}$. A $5.0 \mathrm{GHz}$ single stage low noise amplifier is used in IEEE 802.11a standards for wireless local area network (WLAN). We can use this technology with the CMOS and the latest technology of double-gate MOSFET for designing a RF CMOS switch [24-26]. 
International Journal of VLSI design \& Communication Systems (VLSICS) Vol.3, No.2, April 2012

Table 1. Comparison of LNA performance

\begin{tabular}{|c|c|c|c|c|}
\hline References & Frequency & Gain $\left(\mathbf{S}_{\mathbf{2 1}}\right)$ & $\mathbf{N F}$ & $\mathbf{S}_{\mathbf{1 1}}$ \\
\hline$[19]$ & $5.5 \mathrm{GHz}$ & 10 & 6.2 & -12 \\
\hline$[20]$ & $5.7 \mathrm{GHz}$ & 16.4 & 3.5 & -11 \\
\hline$[21]$ & $5.6-5.8 \mathrm{GHz}$ & 21.4 & 4.4 & Better than 7 \\
\hline$[22]$ & $5.3 \mathrm{GHz}$ & 13 & 2.1 & -13 \\
\hline$[23]$ & $5 \mathrm{GHz}$ & 18.3 & 1.63 & -- \\
\hline This Work & $5 \mathrm{GHz}$ & 25.3 & 2.22 & -12.2 \\
\hline
\end{tabular}

\section{ACKNOWLEDGEMENTS}

The authors would like to thank Prof. G. Singh, Department of Electronics and Communication Engineering, Jaypee University of Information Technology, Solan, India, who permit to take the reference of their work related to Silicon MOSFET Technology. The authors also want to thank Prof. K. S. Yadav, Sr. Scientist, CEERI, Pilani, India for many insightful discussions.

\section{REFERENCES}

[1] Wei Guo and Daquan Huang, "The noise and linearity optimization for $1.9 \mathrm{GHz}$ CMOS low Noise Amplifier," Proc. of IEEE Asia Pacific Conf. on ASIC, Taipei, Taiwan, 6-8 Aug. 2002, pp. 253-257.

[2] Hsieh Hung Hsieh, "A $40 \mathrm{GHz}$ low noise amplifier with a positive-feedback network in 0.18 $\mu \mathrm{m}$ CMOS," IEEE Trans. on Microwave Theory and Techniques, vol. 57, no. 8, pp. 1895-1902, Aug. 2009.

[3] Viranjay M. Srivastava, K. S. Yadav, and G. Singh, "Design and performance analysis of doublegate MOSFET over single-gate MOSFET for RF switch,” Microelectronics Journal, vol. 42, no. 3, pp. 527-534, March 2011.

[4] Nazif Emran Farid, Arjuna Marzuki, and Ahmad Ismat,"A variable gain $2.5 \mathrm{GHz} \mathrm{CMOS}$ low noise amplifier for mobile wireless communications," Proc. of $9^{\text {th }}$ IEEE Int. Conf. of communications, Kuala Lumpur, Malaysia, 15-17 Dec. 2009, pp. 885-889.

[5] Ming Hsien Tsai, S. Hsu, Fu Lung Hsueh, Chewn Pu Jou, Sean Chen, and Ming Hsiang, "A wideband low noise amplifier with $4 \mathrm{kV}$ HBM ESD protection in $65 \mathrm{~nm}$ RF CMOS," IEEE Microwave and Wireless Components Letters, vol. 19, no. 11, pp. 734-736, Nov. 2009.

[6] Bo Huang, Chi Hsueh Wang, Chung Chun Chen, Ming Fong Lei, Pin Cheng Huang, Kun You Lin, and Huei Wang, "Design and analysis for a $60 \mathrm{GHz}$ low noise amplifier with RF ESD protection," IEEE Trans. on Microwave Theory and Techniques, vol. 57, no. 2, pp. 298-305, Feb. 2009.

[7] $\mathrm{Yu}$ Lin Wei and Jun De Jin, "A low power low noise amplifier for K-band applications," IEEE Microwave and Wireless Components Letters, vol. 19, no. 2, pp. 116-118, Feb. 2009.

[8] Bonghyuk Park, Sangsung Choi, and Songcheol Hong, "A low noise amplifier with tunable interference rejection for 3.1 to $10.6 \mathrm{GHz}$ UWB systems," IEEE Microwave and Wireless Components Letters, vol. 20, no. 1, pp. 40-42, Jan 2010.

[9] A. Meaamar, Boon Chirn Chye, Man Anh, and Yeo Kiat Seng, "A 3 to $8 \mathrm{GHz}$ low noise CMOS amplifier," IEEE Microwave and Wireless Components Letters, vol. 19, no. 4, pp. 245-247, April 2009.

[10] Viranjay M. Srivastava, K. S. Yadav, and G. Singh, "Analysis of double gate CMOS for double-pole four-throw RF switch design at 45-nm technology," J. of Computational Electronics, vol. 10, no. 1-2, pp. 229-240, June 2011.

[11] Hyung Jin Lee, Dong Sam Ha, and Sang S. Choi, "A systematic approach to CMOS low noise amplifier design for ultra wideband applications," Proc. of Int. Symp. on Circuit and System, Kobe, Japan, 23-26 May 2005, pp. 3962-3965. 
International Journal of VLSI design \& Communication Systems (VLSICS) Vol.3, No.2, April 2012

[12] Wei Chang Li, Chao Shiun Wang, and Chorng Kuang Wang, "A 2.4 GHz/3.55 GHz/5 GHz multiband LNA with complementary switched capacitor multi-tap inductor in $0.18 \mu \mathrm{m} \mathrm{CMOS,"} \mathrm{IEEE}$ National Science Council (NSC) Taiwan, 2006, pp.1-4.

[13] Roee Ben Yishay, Sara Stolyarova, Shye Shapira, Moshe Musiya, David Kryger, Yossi Shiloh, and Yael Nemirovsky, "A CMOS low noise amplifier with integrated front-side micro-machined inductor," Microelectronics Journal, vol. 42, no. 5, pp. 754-757, May 2011.

[14] Thomas H. Lee, The Design of CMOS Radio-Frequency Integrated Circuits, Cambridge Univ. Press, $2^{\text {nd }} E d ., 2004$.

[15] Nam Jin, "A low power 3.1-10.6 GHz ultra wide band CMOS low noise amplifier with common gate input stage," Current Applied Physics, vol. 11, no. 1, pp. 87-92, Jan. 2011.

[16] Viranjay M. Srivastava, K. S. Yadav, and G. Singh, "Capacitive model and S-parameters of doublepole four-throw double-gate RF CMOS switch," Int. J. of Wireless Engineering and Technology, vol. 2, no. 1, pp. 15-22, Jan. 2011.

[17] H. W. Chiu, "A $2.17 \mathrm{~dB}$ NF $5 \mathrm{GHz}$ band monolithic CMOS LNA with $10 \mathrm{~mW}$ DC power consumption," IEEE Trans. Microwave Theory Tech., vol. 53, no. 3, pp. 813-824, March 2005.

[18] Hsien Chin Chiu, Chia Shih Cheng, Hsuan Ling Kao, Jeffrey S. Fu, Qiang Cui, and Juin J. Liou, "A fully on-chip ESD protection UWB band low noise amplifier using GaAs enhancement-mode dualgate pHEMT technology," Microelectronics Reliability, vol. 51, no. 12, pp. 2137-2142, Dec. 2011.

[19] Yuan Gao, Yuanjin Zheng, and Ban Leong, "A $0.18 \mu \mathrm{m}$ CMOS UWB LNA with $5 \mathrm{GHz}$ interference rejection," IEEE Radio Frequency Integrated Circuits (RFIC) Symp. Honolulu, Hawaii, USA, 3-5 June 2007, pp. 47-50.

[20] Y. S. Wang and L. H. Lu, "5.7 GHz low power variable gain LNA in $0.18 \mu \mathrm{m}$ CMOS," Electronics Letters, vol. 41, no. 2, pp. 66-68, Jan. 2005.

[21] M. Kumarasamy, Chin Boon, and K. Nuntha Kumar, "A fully integrated variable gain 5.75 GHz LNA with on-chip active balun for WLAN," IEEE Radio Frequency Integrated Circuits (RFIC) Symp., 810 June 2003, pp. 439-442.

[22] Seon Myoung, Sang Cheon, and Jong Yook, "Low noise and high linearity LNA based on InGap/GaAS HBT for 5.3 GHz WLAN," European Gallium Arsenide and Other Semiconductor Application Symp. (EGAAS), Paris, 2005.

[23] Hye Ryoung Kim and Sang Gug Lee, "A 5-GHz LNA for wireless LAN application based on $0.5 \mu \mathrm{m}$ SiGe BiCMOS," 2002 IEEE $3^{\text {rd }}$ Int. Conf. on Microwave and Millimeter Wave Technology, 17-19 Aug. 2002, pp. 50-53.

[24] Viranjay M. Srivastava, K. S. Yadav, and G. Singh, "Design and performance analysis of cylindrical surrounding double-gate MOSFET for RF switch," Microelectronics Journal, vol. 42, no. 10, pp. 1124-1135, Oct. 2011.

[25] Mu Chun Wang, Hsin Chia Yang, and Yi Jhen Li, "Minimization of cascade low noise amplifier with $0.18 \mu \mathrm{m}$ CMOS process for $2.4 \mathrm{GHz}$ RFID applications," Electronics and Signal Processing, Lecture Notes in Electrical Engineering, vol. 97, pp. 571-578, 2011.

[26] Viranjay M. Srivastava, K. S. Yadav, and G. Singh, "Possibilities of HfO2 for double-pole four-throw double-gate RF CMOS switch," $4^{\text {th }}$ IEEE Int. Symp. on Microwave, Antenna, Propagation and EMC Technologies for Wireless Communications (MAPE-2011), Beijing, China, 1-3 Nov. 2011, pp. 309312. 
International Journal of VLSI design \& Communication Systems (VLSICS) Vol.3, No.2, April 2012

\section{Authors}

Ravinder Kumar received the Bachelor degree in Electronics and Communication Engineering from Kurukshetra University, Kurukshetra, India in 2009. He is currently pursuing Master degree in Electronics and Communication Engineering at Jaypee University of Information Technology, Solan, India. His research interest includes RFIC design, VLSI design, Networking and Wireless Communication.

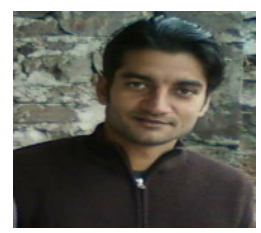

Munish Kumar received the Bachelor degree in Electronics and Communication Engineering from Kurukshetra University, Kurukshetra in 2009. He is currently pursuing Master degree in Electronics and Communication Engineering at Guru Jambheshwar University of Science and Technology, Hisar, india. His research interest includes Networking and Mobile Communication.

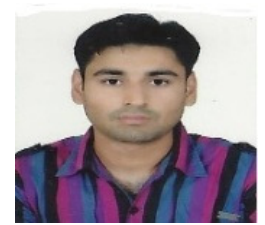

Viranjay M. Srivastava received the Bachelor degree in Electronics and Instrumentation Engineering from the Rohilkhand University, Bareilly, India and the Master degree from VLSI Design Department, Center for Development of Advanced Computing (C-DAC), Noida, India and presently pursuing his doctorate in the field of RF microelectronics. He was with the Semiconductor Process and Wafer Fabrication Center of BEL Laboratories, Bangalore, India, where he worked on characterization of MOS devices, fabrication of devices and development of circuit design. Currently he is a faculty in Jaypee University of Information Technology, Solan, Himachal Pradesh, India. His research and teaching interests includes VLSI design and CAD

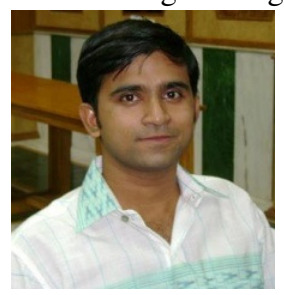
with particular emphasis in low-power design, Chip designing, VLSI testing and verification.He has more than 8 years of teaching and research experience in the area of VLSI Design, RFIC Design, and Analog IC design. He has supervised a number of $\mathrm{B}$. Tech. and $\mathrm{M}$. Tech theses. He is member of IEEE, ACEEE and IACSIT. He has served on the program committee of several seminars, workshops and conferences. He has worked as a reviewer for several conferences and Journals both nationally and internationally. He has published over 50 research papers in different Journals and Conferences and is author of the book, VLSI Technology, Kamal Publishing House, Kanpur, India. 